zu erhalten. Eigenschaften wie Gesichtserkennung und Fotoeffekte sind zwar eine nette Zugabe, aber für die Wahl einer Kamera für Ärzte eher zu vernachlässigen. In jedem Fall sollte die Möglichkeit gegeben sein, dass man Datum und Uhrzeit der Aufnahme auf dem Bild automatisch speichern lassen kann. Funktionen wie diese sollten sich aber einfach an- und wieder abstellen lassen.

\section{Auf das Zubehör kommt es an}

Zur richtigen Kamera gehört das passende Zubehör. Ein Stativ und eine stabile Aufbewahrungstasche sollte sich jeder Arzt gleich mit anschaffen. Hier gibt es Modelle von mehreren Anbietern. Ebenfalls sollte man mehrere Speicherkarten kleiner Kapazität ( 4 bis 8 Gigabyte) mit kaufen - das ist besser als eine große Speicherkarte. Somit geht bei einem möglichen Defekt der Karte nicht der gesamte Inhalt verloren, sondern nur ein Teil. Zusätzlich vermag ein separater
Blitz die Szenerie (das Aufnahmefeld und die Umgebung für das Foto) besser auszuleuchten als der in der Kamera enthaltene. Der Extrablitz muss sich dann aber auch von der Kamera ansteuern lassen. Statt eines Frontalblitzes empfehlen sich für den Praxiseinsatz Ringleuchten, die auf das Objektiv gesteckt werden. Sie leuchten vor allem bei Nahaufnahmen, wie sie Ärzte häufiger anfertigen, die Szenerie gleichmäßig aus. Zur Nachbearbeitung der Bilder liegen den Geräten häufig Programme bei, mit denen sich die Qualität verbessern lässt. Doch mit deren Verwendung sollte man vorsichtig sein, um die eventuelle Beweisfähigkeit eines Fotos (durch Veränderungen oder Manipulationen) nicht zu gefährden. Um die Fotos zu sichern, dürfen sie nicht lange in der Kamera bleiben, sondern alle Bilder sollten auf den Praxiscomputer oder einen separaten Festplattenspeicher übertragen werden.

Thomas Jungbluth

\title{
Digitale Bilder - kleine Helfer im Praxisalltag
}

\section{Ob bei Regressen, der Dokumentation oder im Patientengespräch - Einsatzbereiche für digitale Bilder gibt es viele.}

D ie digitale Fotografie nimmt in der Arztpraxis eine immer wichtigere Rolle ein, zum Beispiel bei der Dokumentation von Behandlungsverläufen. Mit digitalen Bildern lässt sich etwa der Wundstatus bei chronisch venösen Ulcera cruris festhalten und vergleichen. Hier gehört die Fotodokumentation sogar zu den obligaten Leistungsinhalten, um die Behandlung nach EBM-Ziffer 02312 abzurechnen.

Aber auch bei urologischen Befunden hat die Fotodokumentation einen hohen Stellenwert. Beispielsweise lässt sich der Verlauf entzündlicher Veränderungen des äußeren Genitals dokumentieren. Vor allem hier sollte der Arzt darauf achten, dass die Bilder so aufgenommen werden, dass sie später auch miteinander vergleichbar sind. Um Fehler zu vermeiden, sollte immer der gleiche Bildausschnitt gewählt werden und die Belichtung richtig eingestellt sein. Außerdem sollten Ort und Zeitpunkt der Aufnahme festgehalten werden.

Die Bilddokumentation kann den Arzt aber auch vor Regressen schützen. Denn mit einem Foto kann sich etwa die Notwendigkeit einer Therapie belegen lassen. Besonders hilfreich ist das bei der Verordnung von Heilmitteln. Kommt es tatsächlich zu einer Regressforderung, können Ärzte mit den digitalen Bildern eine durchgehende Verlaufsdokumentation vorlegen. Das erleichtert ihnen Argumentation und Beweisführung. Ein weiterer Vorteil der digitalen Bilder ist, dass die Fotos schnell und unkompliziert per EMail an einen Fachkollegen oder eine Klinik gesendet werden können.

Fotos eignen sich als gute Stütze fürs Patientengespräch, etwa um Patienten den Verlauf einer Erkrankung auch visuell aufzuzeigen. In heiklen Fällen, wie dem Verdacht auf Misshandlung, kann die Kamera ebenfalls eingesetzt werden. Verletzungen etwa bei Kindern können zur Beweissicherung fotografiert werden. Bei Minderjährigen müssen jedoch die Eltern vorab ihr Einverständnis geben. $\quad$ mn
Befunde dreidimensional dokumentieren

Panasonic plant die Entwicklung des nach eigenen Angaben weltweit ersten Doppel-Objektivs für 3D-Aufnahmen mit digitalen Wechselobjektivkameras. Das Objektiv, das in die Produktreihe Lumix G Micro System aufgenommen wird, ermögliche die Aufnahme von Porträt- und Landschaftsaufnahmen, aber eben auch von weiteren Motiven in 3-D-Qualität. Gerade Letzteres könnte das Objektiv auch für Ärzte interessant machen. Allerdings lassen sich die 3-D-Bilder - zumindest in dreidimensionaler Form - nur über einen 3-D-fähigen Bildschirm ansehen.

reh

\section{Zubehör für schattenfreie Nahaufnahmen}

Gerade bei Nahaufnahmen kann der Einsatz eines Blitzes eher hinderlich als hilfreich sein. Eine gleichmäßige und schattenfreie Ausleuchtung gelingt etwa mit dem LED-Ringlicht von Dörr, das sich laut Hersteller besonders für Aufnahmen im Medizinbereich eignet. Es kann auf digitale Spiegelreflex- und Kompaktkameras aufgesetzt werden und beinhaltet in der einfachen Version zwölf LEDLampen. Ein integrierter Akku erlaubt auch den mobilen Einsatz. Kosten soll das Ringlicht 139 EUR. reh

\section{Wie auch kleine Bilder an Größe gewinnen}

Es passiert schon einmal im hektischen Praxisalltag: Man war zu weit weg vom Fotoobjekt und jetzt ist die Aufnahme zu klein. In einem solchen Fall können spezielle Computer-Programme helfen, das Bild ohne dass es an Schärfe verliert - zu vergrößern. Ein Beispiel ist der Akvis Magnifier. Dank eines effektiven Algorithmus, so der Hersteller Akvis, könne das Programm digitale Bilder zu sehr hohen Auflösungen vergrößern (bis hin zum Posterformat mit $30.000 \times 30.000$ Pixeln). Ärzte sollten allerdings beachten, dass jede Nachbearbeitung die Beweiskraft von Bildern gefährdet. Deshalb: Immer auch das Original speichern. reh 